\title{
Lane Departure and Collision Controlling using Image Processing
}

\author{
Shambhavi Hiremath, Vasanta Koti, S. V. Viraktamath
}

\begin{abstract}
Lane departure is an Advanced Driver Assistance System (ADAS). In today's world of technology, the automotive sector is gaining high demand. The advancements in this field have made life easy and danger free. Road accidents lead to large set of accidents because of the negligence of driver hence an automatic system has to be designed. Here, road lane detection, tracking method and warning alert system is proposed and implemented using MATLAB software. This paper displays the warning message for each departure of the vehicle and thus avoiding the collision.
\end{abstract}

Keywords: Hough transforms, Lane detection, Lane tracking, Thresholding.

\section{INTRODUCTION}

Road accidents lead to thousands of causalities every year. Driver Assistance Systems (DAS) is used to avoid the most of these accidents caused by driving errors. A variety of DAS exists that aim to improve road and driver safety. One of these systems is the lane departure warning system that uses a road lane detection algorithm to detect if car departs from the lane. Today lane departure warning systems only notify the driver and do not intervene, but their role becomes more active in the future as the automation of driving progresses. The increasing responsibility poses new challenges to DAS. They need to be more reliable, more robust and react even faster. Consequently, it will be demanded to lane departure warning systems that they detect a departure from a lane quick enough to be clever to counteract it. To do so, algorithms for road lane detection are necessary that deliver very accurate and fast estimates of lane positions. However, recent developments have made other processing units such as General-Purpose Units (GPU) and more importantly, Field Programmable Gate Arrays (FPGA) a feasible alternative or complement to Engine Control Unit (ECU). Especially FPGAs promise faster and more flexible processing at less power consumption.

Revised Manuscript Received on October 30, 2019.

* Correspondence Author

Shambhavi Hiremath, Electronics and Communication, S.D.M. College of Enginnering and Technology, Dharwad, India Email:hshambhavi07@gmail.com

Vasanta Koti, Electronics and Communication, S.D.M. College of Enginnering and Technology, Dharwad, India Email:vasantakoti128@gmail.com

Dr. S. V. Viraktamath, Electronics and Communication, S.D.M. College of Enginnering and Technology, Dharwad, India

Email: svvitc2011@gmail.com

(C) The Authors. Published by Blue Eyes Intelligence Engineering and Sciences Publication (BEIESP). This is an open access article under the CC BY-NC-ND license (http://creativecommons.org/licenses/by-nc-nd/4.0/)
A lane departure warning system can be defined as a mechanism modeled to alert the driver when the vehicle starts to move out of its lane on arterial roads and freeways. The accidents due to distractions, driver error and drowsiness can be reduced. The accidents on the road has lead the ongoing technologies to design a model for the road system which prevents the drivers to cross the lane if the separation between two vehicles is less or at the joining of two roads hence a lane departure systems are developed to prevent the vehicles collision. The lane has to be detected first and then that has to be tracked so as to check the vehicle's movement along the defined lane. After tracking the vehicle if it crosses the lane necessary warning messages are displayed.

\section{RELATED WORK}

Several models for lane boundaries have been proposed. A lane tracking system through particle filtering is studied in [1]. For approximating lane boundaries, parabolic models are used in color video sequences is discussed in [2]. Lane boundaries can be detected using Computer vision and Time for Lane Crossing (TLC) can be estimated [3]. Along with this, radar-like information can also be used to estimate TLC as discussed in [4]. The lane orientation is estimated through an Edge Distribution Function (EDF) and the vehicle direction is identified to find any changes [5]. Lane boundaries detection through a linear-parabolic model is studied in [6]. Sen-Ching S. Cheung and Chandrika Kamath [7] suggested background subtraction, which finds moving objects by subtracting the current frame from a background. To improve background subtraction and object segmentation the gray color information is used [8]. The background subtraction with less computational complexity is proposed in [9]. The two different techniques, a Background Subtraction and two frame difference technique are used for detecting the moving objects [10]. Video to frame conversion of Television news video by using MATLAB is discussed in [11]. In [11], two steps for lane detection can be explored. Firstly, pre-processing and secondly lane marking. Pre-processing includes steps like Region of Interest [ROI] selection, gray scaling, edge detection and defining threshold value. For video, frames will be extracted and background subtraction is performed to track the lane. The present work is aimed at the use in a future DAS, a time critical application, and should therefore deliver its results as fast as possible. 


\section{METHODOLOGY}

In first step, the algorithm pre-processes the incoming frames to emphasize relevant information. Once the processing of frames is over, each frame undergoes Hough transform to detect line markings and displaying the warning message if the vehicle crosses the lane. Below figure is the block diagram of the proposed system.

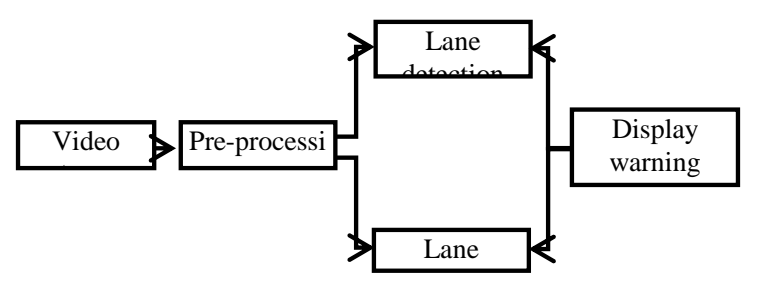

Fig. 1 Block diagram of proposed system

\section{A.Pre-processing Stage}

The pre-processing involves following steps: ROI selection, gray conversion, edge detection and thresholding.

\section{a. Region of Interest (ROI) Selection}

The region that contains the essential information is selected and the remaining part is discarded. The ROI selection is before segmentation. The ROI removes the major noise contents present in the image.

\section{b. Gray Scaling}

The ROI can be transformed to a gray scale format, where each pixel reflects the pixel intensity present in the original image. Dark pixel will receive low intensity values and bright pixels will receive high values. The RGB to gray scale conversion is done on MATLAB.

\section{c. Edge Detection}

A canny detector is applied to the image to detect the edges in the image. Initially a Sobel filter was used for this reason but the lines were not correctly observed. Each pixel in the new image describes the gradient of the original image at that position. The gradient values are calculated using the mathematical equations.

\section{d. Thresholding}

Some disturbances will only create thin and weak edges. These disturbances can be deleted by thresholding. The intensity of all pixels, whose gradient falls below a certain threshold, is set to zero. Since the lane markings receive high gradients by the Sobel filter, there will not be any negative affect.

\section{B. Video Processing}

Video signal is a sequence of images with varying time. The spatial distribution of intensities remains constant with time in still image, whereas it varies with time in time varying image. Frame extraction comes first in video processing. Pre-processing is the next stage after the frames are extracted. Pre-processing follows below steps:

\section{a. Frame Extraction}

Frame extraction which extracts the frames from the video. A set of frames forms the shots and the group of shots will produce the scenes. The video is a combination of these scenes.

\section{b. Pre-processing}

The steps of pre-processing are explained in earlier sections. The pre-processing mainly involves converting an image into gray then applying an edge detection algorithm followed by thresholding to remove the negligible pixel values

\section{c. Background Subtraction}

This technique is used for motion segmentation in static images. It uses the technique of subtracting the current image pixel-by-pixel from a background image to find movement of the object.

\section{d. Hough Transform}

Here, algorithm uses an accumulator (2D array), to detect the existence of a line described by $r=x \cos \theta+y \sin \theta$. The number of unknown parameters (quantized values of $r$ and $\theta$ in the pair $(r, \theta))$ decides the dimension of the accumulator. For each pixel at $(\mathrm{x}, \mathrm{y})$ and its neighbourhood, the Hough transform algorithm is used to determine the sufficient evidence of a straight line at that pixel. If so, it will calculate the parameters $(r, \theta)$ of that line, and then look for the accumulators bin that the parameters fall into and increment the value of that bin. Once the parameters are obtained, Hough peaks are determined and next hough lines are obtained based on these peak values. Fig. 2 describes the flow chart of the lane departure warning system.

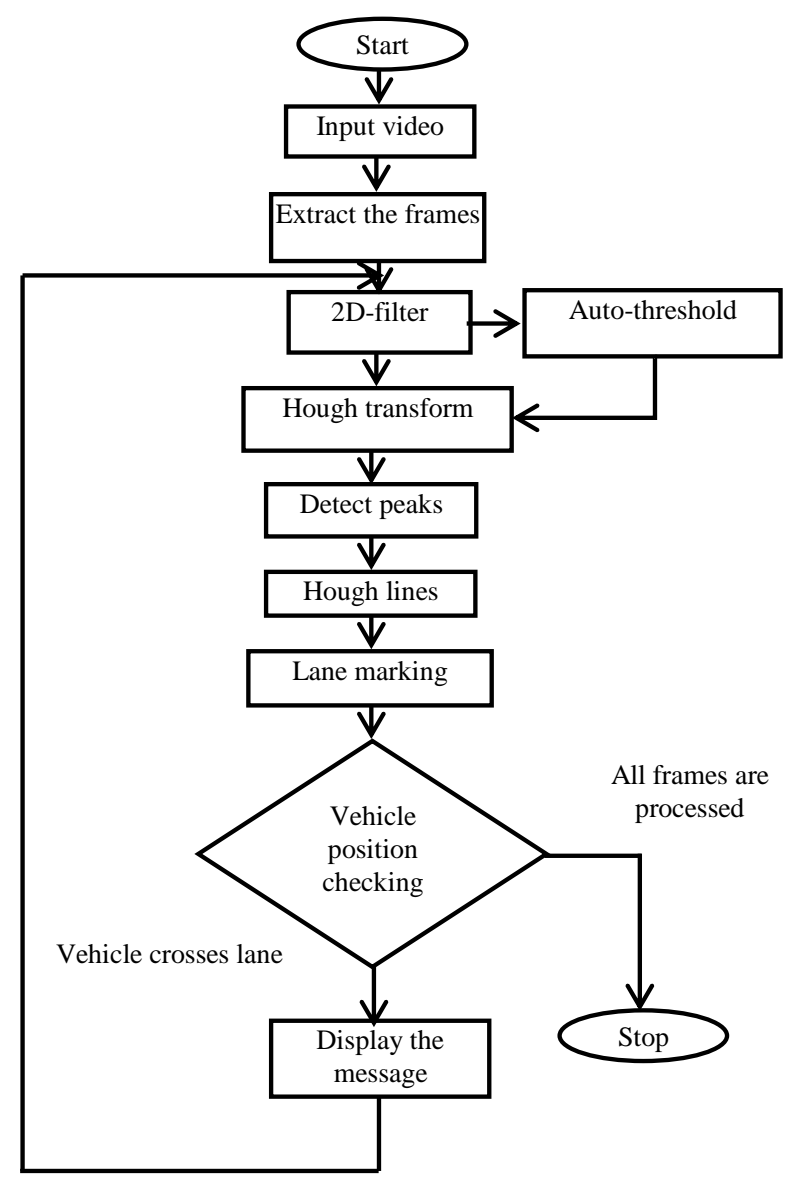

Fig. 2 Flow chart of lane departure warning system

\section{Display Lane Departure Warning Message}

Once the variables required are initialized, each frame is taken and it is compared with corresponding previous frame. When the frame is greater than 5 then only the offset is added else not and if there is some difference 
between the previous and current frames then the suitable warning message will be displayed. For every frame the background subtraction is applied and the output message is displayed. To indicate the region of interest Shape Inserter function is used. For detecting the lane markers the shape used is 'lines' and for indicating the region shape used is 'polygon'. The process is repeated until the driver changes the direction of vehicle. The final video is displayed in steps of RGB.

\section{RESULTS AND DISCUSSION}

The following are the results based on simulation done by using two filters. Canny and Sobel are the two filters which are used and compared with the output obtained.

\section{A. Results Considering Canny Filter}

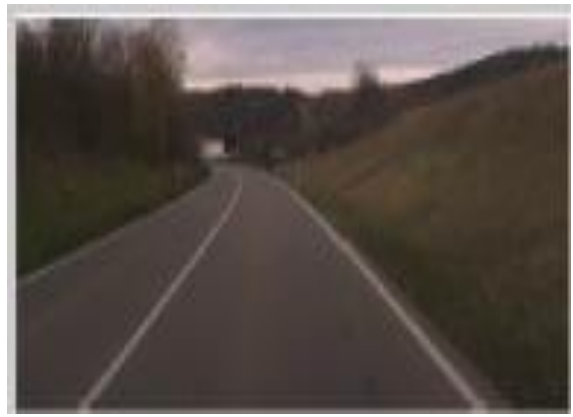

Fig. 3 Original image

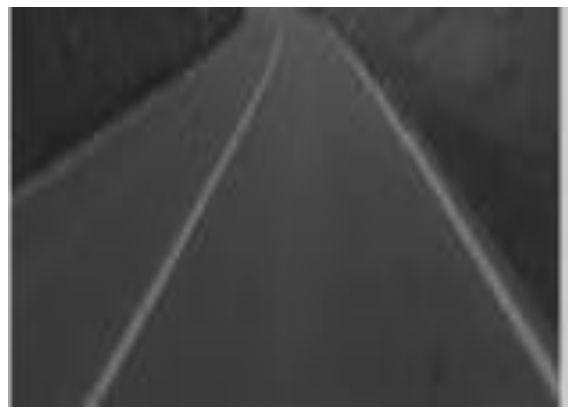

Fig. 4 Gray image

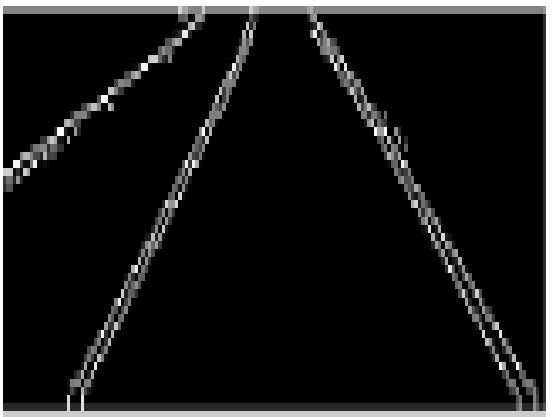

Fig. 5 Canny filter with threshold 0.09

Initially as from Fig. 3, the original image of the lane is considered in which only region of interest is taken for further processing operations using the appropriate filters. As shown in Fig. 4, gray scale image of the original image is preferred because in cases like finding the edges or other features where there is step change in pixel value Gray scaling is better than color. As shown in Fig. 5, it indicates the result of filtering the gray scale image using canny filter. The threshold value set in this case is $\mathrm{T}=0.09$.

\section{B. Results for Sobel filter with Different Threshold Values}

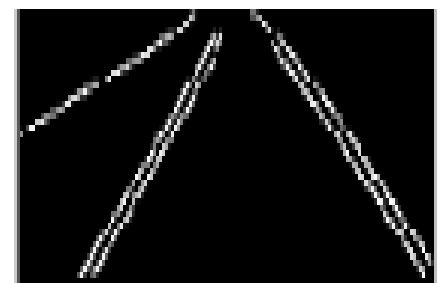

Fig. 6 Image with threshold=0.09

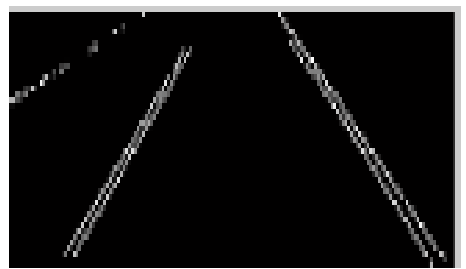

Fig. 7 Image with threshold $=\mathbf{0 . 1 6}$

As shown in Fig. 5, it is the result of using Sobel filter for filtering operation. Here the threshold value set is 0.09 . It can be clearly identified that the edges are at the required region, where the lane is clearly detected. As shown in Fig. 6, it is the result of simulation using Sobel filter with threshold value set as $\mathrm{T}=0.16$. As compared to this figure, the output with $\mathrm{t}=0.16$ is more accurate in lane detection but only dominant pixels will be detected.

\section{Results for Edge Detected Binary Image using Binary Subtraction}

Case 1: Images without shadow:

In this case we consider the road without suffering from the shadow of the surrounding environment.

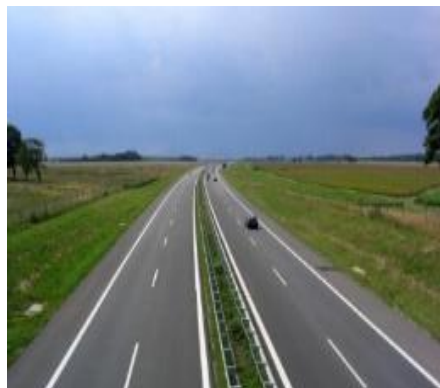

(a)

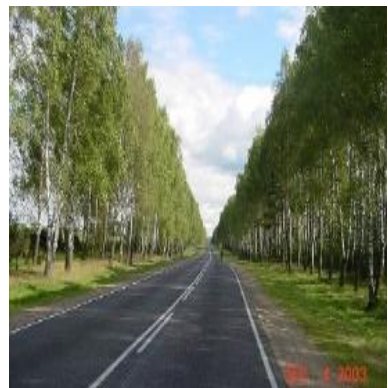

(b)

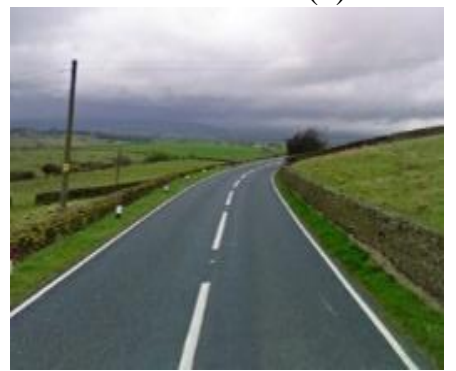

(c)

Fig. 8 Original images set 1 


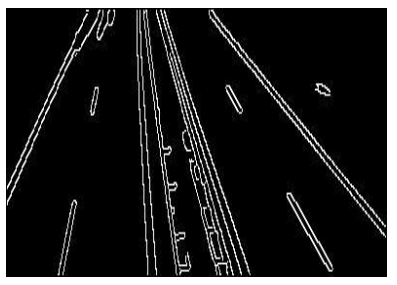

(a)

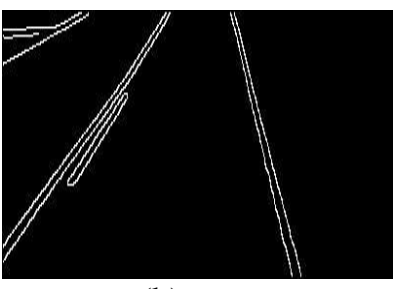

(b)

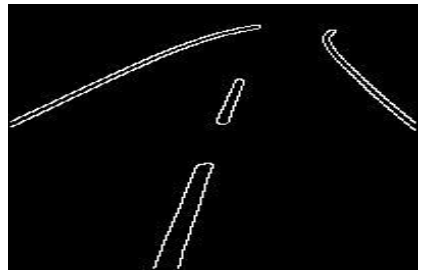

(c)

Fig. 9 Detected edges of original image set 1

As shown in (a), (b) and (c) of Fig. 9, it is image in which edge is detected using background subtraction method. Original image is converted into binary image, then applied canny filter with threshold 0.41 . Then this image is subjected to background subtraction to detect the edges.

Case 2: Images with shadow:

In this case we consider the road which is prone to shadow from the surrounding environment

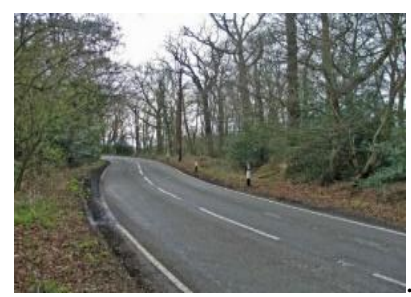

(a)

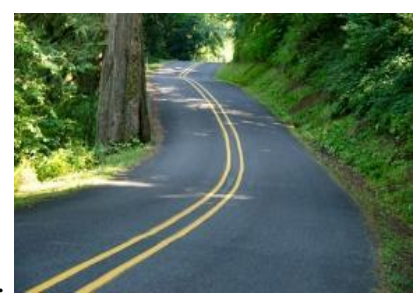

(b)
Fig. 10 Original images set 2

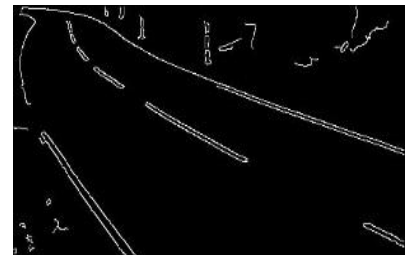

(a)

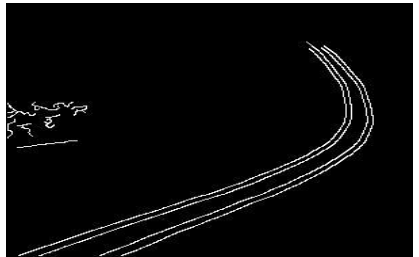

(b)
Fig. 11 Detected edges of original image set 2

As shown in (a) and (b) of Fig. 10, these are the original images of the road which are prone to shadow from the surrounding environment. As shown in (a) and (b) of Fig. 11, these are the edge detected images of Fig. 10. In this method the basic components of each image such as blue, red and green are extracted and considering the components of color present in the shadow, color constituents are subtracted.

\section{Results of Frames Extraction}

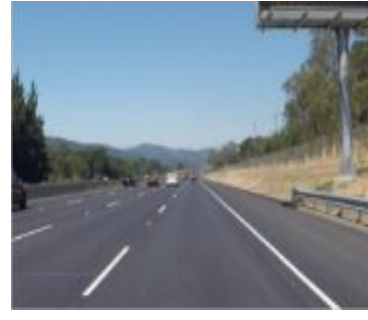

(a)

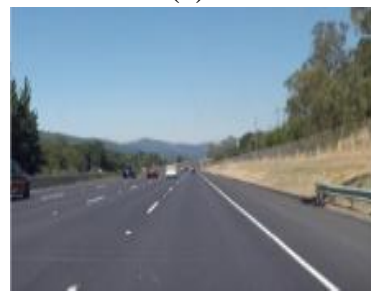

(c)

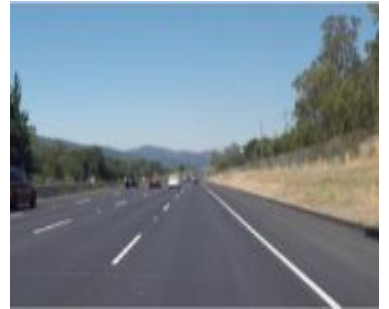

(d)

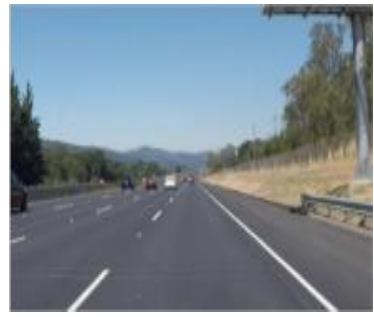

(b)

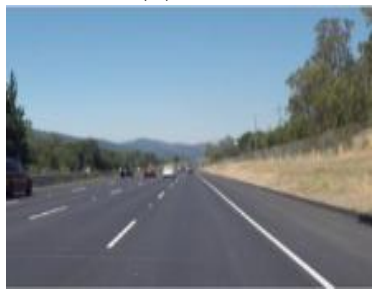

(d)

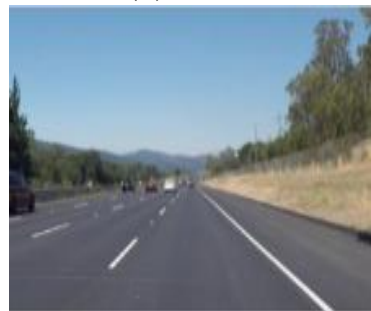

(f)
Fig. 12 Extracted frames from video

As shown in Fig. 12, these are the extracted images from the video in the order (a) to (f) with 1 second of interval between each frame.

\section{E. Results of Cropped and Edge Detected Frames}

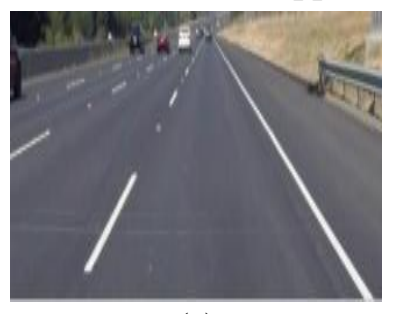

(a)

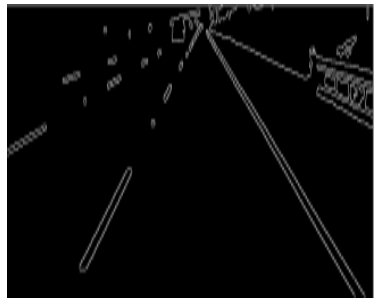

(c)

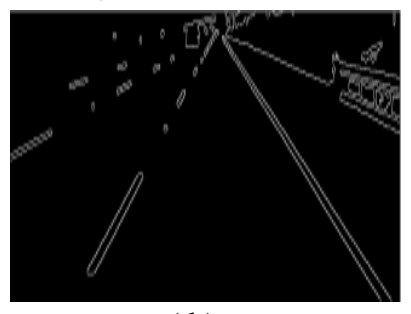

(b)

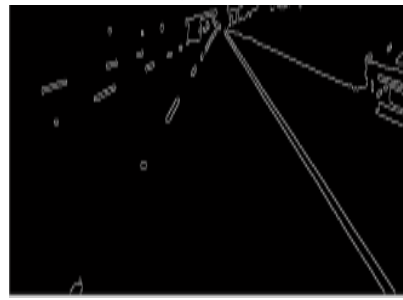

(d)
Fig. 13 Edge detected images of extracted frames 


\section{F. Results of Lane Departure Warning Issued}

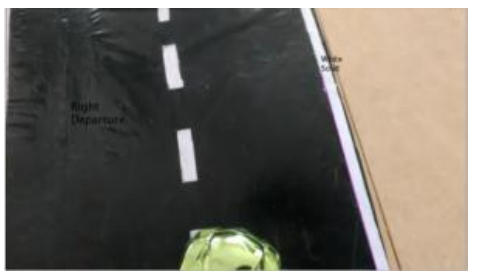

Fig. 14 Initial position of the vehicle towards right

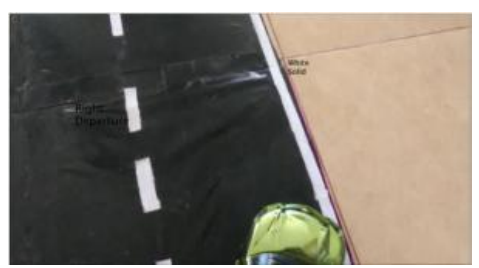

Fig. 15 Vehicle departing

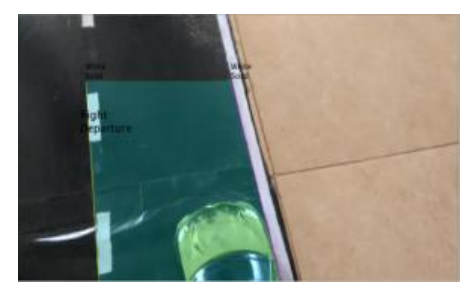

Fig. 16 Vehicle along the right lane towards left

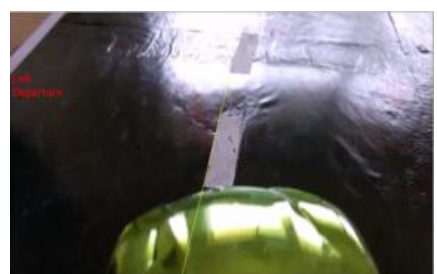

Fig. 17 Vehicle departing

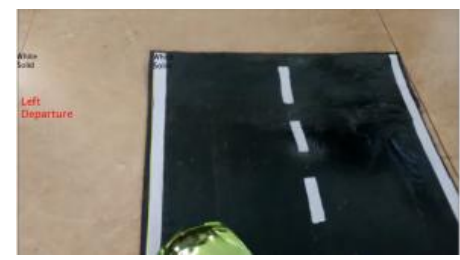

Fig. 18 Vehicle about to depart from left lane

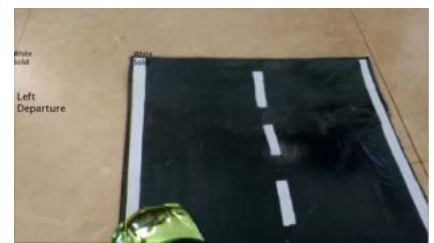

Fig. 19 Vehicle moving away from left lane

\section{CONCLUSION}

A lane departure warning system is developed and results are obtained using MATLAB. The incoming video signal can be processed in two ways: the first step is pre-processing wherein the noise is removed while in second step the lane is detected and tracked. The lane detection is employed using edge detection algorithms while the tracking is done using Hough transform. The next step is to issue a warning message in case the driver crosses the lane. The departure is determined by background subtraction principle. The lane markings and the region of vehicle is determined by some functions. The warning message is displayed for each movement of vehicle. Once all the frame processing is done, the output video is displayed. Hence the driver is alerted on crossing the lane and thus preventing the collision of the vehicle.

\section{REFERENCES}

1. N. Apostoloff and A. Zelinsky.: Robust Based lane Tracking using Multiple Cues and Particle Filtering (2003) 558-563

2. M. A. Sotelo, F. J. Rodriguez, L. Magdalena, L. M. Bergasa, and L. Boquete.: AColourvision-based ane Tracking System for Autonomous Driving on Unmarked Roads (2004) 95-116

3. D. J. LeBlanc, P. J. V. G. E. Johnson, G. Gerber, R. D. Sonia, R. D. Ervin, C.-F. Lin, A. G. Ulsoy and T. E. Pilutti.: CAPC: A Road-departure Prevention System (1996) 61-71

4. R. Risack, N. Mohler, and W. Enkelmann.: A Video-based Lane Keeping Assistant (2000)506-511

5. J. W. Lee.: A Machine Vision System for Lane-departure Detection (2002) 52-78

6. C. R. Jung and C. R. Kelber.:A Lane Departure Warning System based on a Linear-Parabolic Lane Model (2004)891-895

7. Sen-Ching S. Cheung and Chandrika Kamath.: Robust Techniques for Background Subtraction in Urban Traffic Video

8. Mahamuni P. D, R. P. Patil.: Moving Object Detection using Background Subtraction Algorithm using Simulink (2014)

9. Leva, J. L. \& Mitre Corporation.: A Fast Normal Random Number Generator(1992)449-453

10. Mrs. Ayeesha Naaz and Mohd Nayeem Uddin.: Moving Object Detection Based on Background Subtraction \& Frame Differencing Technique (2016)

11. Punith Kumar M B, Dr. P.S. Puttaswamy.: Video To Frame Conversion Of TV News Video by using Matlab (2014.

\section{AUTHORS PROFILE}

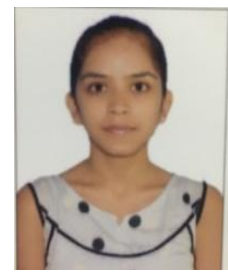

Shambhavi Hiremath student of SDMCET Dharwad, Karnataka, India. In the Department of E\&CE. Certified in HTML course and done project on Simulation of Local Area Network. Research interests in Computer Networking, Image processing and Analog communication.

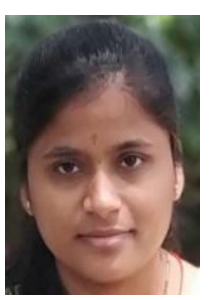

Vasanta Koti graduated in SDMCET, Dharwad, Karnataka, India. In the Department of E\&CE. Research interests in Internet of Things (IoT), Matlab projects, Image Processing and Communication.

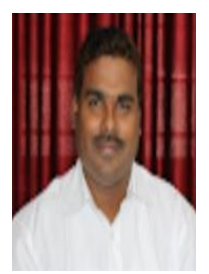

Prof. S. V. Viraktamath is with SDMCET, Dharwad, Karnataka, India. Serving as Assistant Professor (S.G) in the Department of E\&CE. He has received a gold medal from VTU Belgaum for securing first rank in M.Tech (DC\&N). Research interests include Error control coding, Wireless communication and Networking.

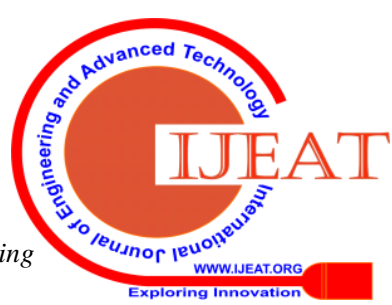

